\title{
Lithographically fabricated optical cavities for refractive index sensing
}

Mark Adams

ENSCO Inc., 4849 North Wickham Road, Melbourne, Florida 32940

Guy A. DeRose ${ }^{\text {a) }}$

Elcetrical Engineering and Applied Physics Department, California Institute of Technology, 1200 East California Boulevard, Pasadena, California 91125

Marko Loncar

Division of Engineering and Applied Science, Harvard University, 9 Oxford Street, Cambridge, Massachusetts 02138

Axel Scherer

Electrical Engineering and Applied Physics Department, California Institute of Technology, 1200 East California Boulevard, Pasadena, California 91125

(Received 3 June 2005; accepted 26 September 2005; published 7 December 2005)

Since the development of distributed Bragg gratings, high resolution lithography and etching have been applied towards the concentration of light. The most important application of lithographically fabricated microcavities has been for the spectral control over laser emission. Here we describe the opportunities that arise from further miniaturization of laser cavities by using high index contrast photonic crystal mirrors and annular Bragg reflectors. We have used these optical cavities, with mode volumes as small as $10^{-17} 1$, to perform spectroscopic analysis and compare the mode volumes and sensitivities of these geometries. (C) 2005 American Vacuum Society. [DOI: 10.1116/1.2127945]

\section{INTRODUCTION}

The construction of compact spectroscopic tools for the optical analysis of ultrasmall $\left(<10^{-15} 1\right)$ sample volumes remains an important goal in the development of integrated microfluidics systems. Miniaturization of appropriate light sources and detectors enables very compact and versatile "laboratory on a chip" devices, in which many analytical functions can be monolithically combined. Porous cavity designs [Figs. 1(a) and 1(b)] permit the introduction of analyte directly into the high optical field of laser cavities. Moreover, due to the ultrasmall mode volume of nanocavity lasers, the detection to optical changes within subfemtoliter volumes becomes possible. The introduction of absorbing or fluorescing molecules into such cavities has a large influence on the optical signature, and the high fields obtained in the cavity can be used for spectroscopy on the cavity contents. This ultimately may enable the sensing and analysis of individual organic molecules or self-assembled quantum dots, and offers a unique opportunity to achieve strong interaction between light and molecules on a nanoscale level. By combining many of these lasers, each with a different peak wavelength, compact spectroscopy tools can be defined. Here we compare two microfabricated laser geometries, the planar photonic crystal (PPC) laser and the annular Bragg resonator $(\mathrm{ABR})$ that combine high refractive index contrast with gain, and that are ideally suited for ambient refractive index sensing.

\footnotetext{
a) Author to whom correspondence should be addressed; electronic mail:
} derose@caltech.edu

\section{NANOCAVITY DESIGNS}

The high- $Q$ cavity geometries are based on fractional edge dislocations in the single defect triangular lattice photonic crystal ${ }^{1}$ and the annular Bragg reflector. ${ }^{2}$ The planar photonic crystal is based on a free-standing membrane: high dielectric constant slab (refractive index $n=3.4$ ) is perforated with two-dimensional 2D lattice of holes with periodicity $a$ and is suspended in the air. The cavity consists of a defect hole (radius $r_{\text {def }}$ ) that is smaller than surrounding holes (radius $r$ ) which define the photonic crystal mirror. The row that contains the defect hole is elongated by moving two photonic crystal half planes a fraction of a lattice constant apart in the $\Gamma X$ direction in Figs. 1(c) and 1(d). Each half plane is moved by $p / 2$, yielding total dislocation of $p$.

It was shown that in such photonic crystal cavity, with $r / a=0.275, r_{\mathrm{def}} / a=0.2$, and $d / a=0.75$ ( $d$ is thickness of the slab), it is possible to achieve $Q$ factors as high as 11000 by tuning the dislocation parameter $p$. These high $Q$ values were obtained while maintaining a very small mode volume of $V_{\text {mode }} \approx 0.1(\lambda / 2)^{3}$. ${ }^{1}$ However, it is clear that the presence of a hole at the point of maximum field intensity is not desirable in low-threshold laser designs, since the overlap with the gain region, provided by quantum wells is decreased. In most common applications, planar photonic crystals are in the form of a free standing membrane suspended in the air. However, it is also of interest to explore the PPC properties when the air is replaced with material with a refractive index greater than 1. This is the case when PPC lasers are used as chemical sensors ${ }^{3}$ and tunable filters in which case PPC cavities are backfilled with electro-optic or nonlinear polymer in order to change their resonant frequency by applying external electromagnetic fields. 

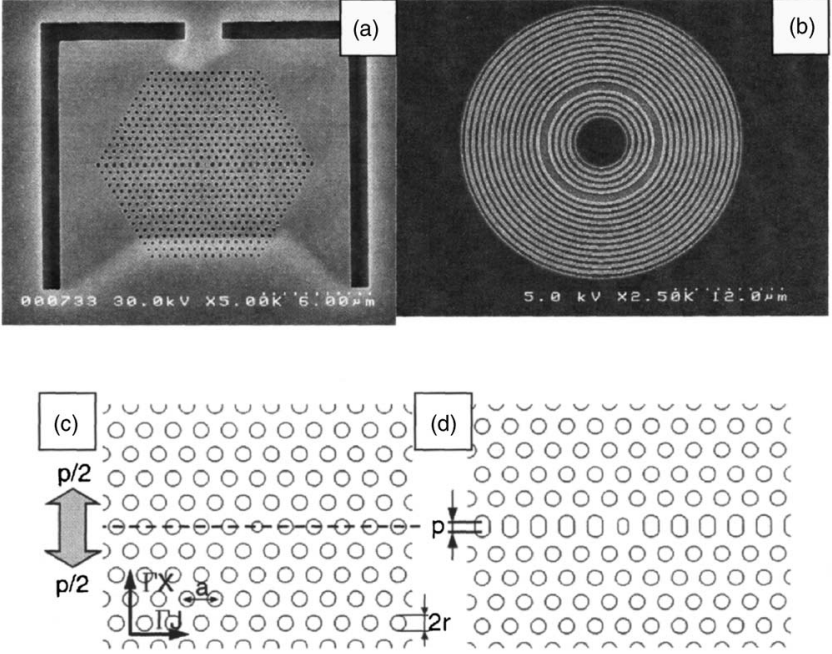

FIG. 1. (a) SEM micrograph of high- $Q$ planar photonic crystal; (b) annular Bragg reflector cavities, defined in thin InGaAsP membranes; and (c) conventional single-defect cavity $(p=0)$. When structure is "cut" along the dashed line, and two PPC half planes are dislocated along $\Gamma X$ direction by $p / 2$ and (d) high- $Q$ cavity can be formed.

In Fig. 2(c) one can see the dependence of the dielectricband and air-band edges when a PPC is immersed in materials with various refractive indices. The thickness of the PPC is $d / a=0.75$ and its refractive index $n_{\text {slab }}=3.4$. The hole size is $r / a$. As expected, the band edges experience redshift when the PPC slab is immersed in a material with higher refractive index. Also, the width of the band gap decreases when $n_{\text {env }}$ increases. The bottom of the air band is more affected with increased $n_{\text {env }}$, since air-band modes have larger spatial overlap with the environment. From Fig. 2(c) one can deduce that the bandgap will close when $n_{\text {env }} \approx 2$ in both structures. In the case of a crystal with $r / a=0.4$ the dependence of the band edges on $n_{\text {env }}$ can be well approximated with ${ }^{4}$

$$
\begin{aligned}
& \frac{a}{\lambda_{\text {dielectric }}}=0.0289 n_{\mathrm{env}}+0.3255, \\
& \frac{a}{\lambda_{\text {air }}}=0.2139 n_{\mathrm{env}}+0.6748 .
\end{aligned}
$$

When $r / a=0.3$, band edges can be expressed as

$$
\begin{aligned}
& \frac{a}{\lambda_{\text {dielectric }}}=-0.0104 n_{\mathrm{env}}+0.2621, \\
& \frac{a}{\lambda_{\text {air }}}=-0.0104 n_{\mathrm{env}}+0.4144 .
\end{aligned}
$$

As expected, the structure with bigger holes is more sensitive to the changes in the refractive index of environment. From these equations one finds that the sensitivity of the air-band edge is better than $\Delta \lambda \approx 700 \Delta n$, when $r / a=0.4$. This suggests that by monitoring the shifts in the emission wavelength of a band-edge laser (that operates at the airband edge) very small changes in the refractive index of the (a)
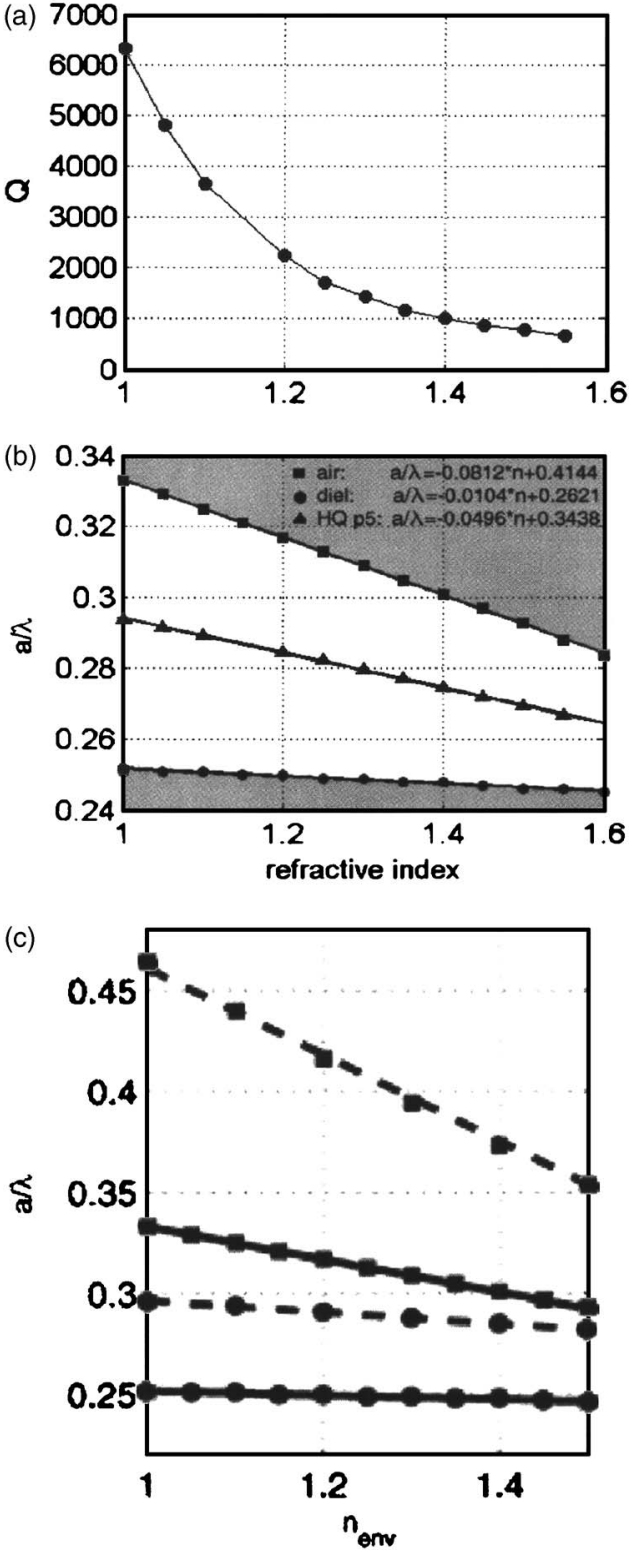

FIG. 2. Dependence of the: (a) $Q$ factor and (b) eigenfrequency of cavity resonance on the refractive index of analyte introduced in the cavity with $p / a=25 \%$. (c) Air band and dielectric band edge dependence on the refractive index of environment $\left(n_{\text {env }}\right)$ surrounding PPC slab, for $r / a=0.3$ (solid lines) and $r / a=0.4$ (dashed lines).

ambient could be detected. A similar approach can be applied in annular Bragg geometries [Fig. 1(b)], where $\Delta \lambda$ $\approx 400 \Delta n$ values have been demonstrated. ${ }^{2}$ Depending on the sensor application, the refractive index sensitivity can be traded off against the analyte volume to be examined.

It is also of interest to determine the change in the $Q$ and eigenfrequency of the modes supported in the cavity based on fractional edge dislocations as the cavity is backfilled with chemicals with higher refractive index $(n>1)$. In Fig. 2(a) it is observed that the highest $Q$ that one could hope to achieve in the modeled cavity design occurs at an ambient refractive index $n_{\mathrm{env}}=1$ (air), and this value deteriorates as the refractive index of the ambient surrounding the photonic 
crystal cavity is increased. This decrease in $Q$ is a result of the weaker vertical confinement of light by total internal reflection, and can be compensated for by increasing the thickness of the photonic crystal slab.

It is interesting to note that the frequency of the resonant mode also depends linearly on the refractive index of the environment $\left(n_{\text {env }}\right)$ [Fig. 2(b)]. From linear fits of the dependence of the resonant frequency on $n_{\text {env }}$, one can estimate the sensitivity of the cavity, and find that the wavelength shift of the resonance is approximately $\Delta \lambda \approx 266 \Delta n_{\mathrm{env}}$, where $\Delta n_{\mathrm{env}}$ is the change in refractive index. The simplest method of optically sensing ambient material uses wavelength shifts in the laser spectrum when the laser is immersed into a solution or exposed to a material to measure its refractive index. In this method, the sensitivity of the sensor depends on the smallest change in refractive index that can be optically detected. In passive devices, this is related to the width of the cavity resonance peak, which in turn is determined by cavity quality $Q$. If one assumes that the cavity is embedded in a typical polymer $\left(n_{\mathrm{env}}=1.4\right)$ a wavelength shift that is still observable from cavity with $Q=1000$ is $\Delta \lambda=1.55 \mathrm{~nm}$, which corresponds to change in refractive index of $\Delta n \approx 0.0056$. On the other hand, once optical gain is introduced into the cavity the linewidth of emission is significantly narrowed $(\Delta \lambda$ $\approx 0.12 \mathrm{~nm}$ ), and sensitivities to $\Delta n<0.001$ can be measured even in cavities with modest $Q$ factors. Figures 2(a) and 2(b) suggest that the edge of the air band is even more sensitive to changes in the refractive index than the cavity mode itself. Therefore, band-edge lasers ${ }^{5-8}$ and ABR lasers ${ }^{2}$ might be even better choice for applications where high sensitivity to the changes of refractive index are needed. However, bandedge lasers operate at extended bulk PPC modes, with large mode volumes, and therefore are not suitable for applications where high spatial sensitivity is needed (e.g., in singlemolecule detection).

The field strength in the central defect hole of the cavity can also be estimated. The energy stored in the resonator can be written as

$$
W_{\text {stored }}=P Q_{\text {env }} \frac{\lambda}{2 \pi c},
$$

where $Q_{\text {env }}$ is the $Q$ factor of the cavity immersed in the environment with refractive index $n_{\text {env }}$. Normalized energy of the cavity mode $\left(K_{\text {env }}\right)$ can be written as

$$
K_{\mathrm{env}}=\frac{\iiint \frac{1}{2} \varepsilon|E|^{2}+\frac{1}{2} \mu|H|^{2} d V}{\varepsilon_{\mathrm{def}}\left|E_{\mathrm{def}}\right|^{2}\left(\frac{\lambda}{2}\right)^{3}}=\frac{W_{\text {stored }}}{\varepsilon_{\mathrm{env}} \varepsilon_{0}\left|E_{\mathrm{def}}\right|^{2}\left(\frac{\lambda}{2}\right)^{3}}
$$

and depends on the environment that the cavity is immersed into. The $E_{\text {def }}$ is the maximum electric field strength in the hole at the center of the cavity. It is important to remember that the resonator can be embedded in materials different from air, and therefore one has to use appropriate permeability of the material for $\varepsilon_{\text {def }}$. Combining these equations, the maximum field strength at the center of the cavity can be expressed as

$$
E_{\mathrm{def}}=\left(\frac{4}{\lambda n_{\mathrm{env}}}\right) \sqrt{\frac{P}{4 \pi \varepsilon_{0} c}} \sqrt{\frac{Q_{\mathrm{env}}}{K_{\mathrm{env}}}} .
$$

From calculations of the dependence of $E_{\mathrm{def}}$ on refractive index of the environment $n_{\mathrm{env}}$, we predict that the maximum field is on the order of the breakdown strength of air, or $E_{\text {breakdown }}=3 \mathrm{~V} / \mu \mathrm{m}$.

\section{FABRICATION PROCEDURE}

InGaAsP quantum well material was grown on InP substrate using metalorganic chemical vapor deposition. Optical gain was provided by four $9 \mathrm{~nm}$ thick, compressively strained, quantum wells with an electronic band gap at $\lambda_{\mathrm{bg}}$ $=1.55 \mu \mathrm{m}$, separated by $20 \mathrm{~nm}$ thick InGaAsP barriers $\left(\lambda_{\mathrm{bg}}\right.$ $=1.22 \mu \mathrm{m})$. This active material was placed in the center of a $330 \mathrm{~nm}$ thick InGaAsP slab $\left(\lambda_{\text {bg }}=1.22 \mu \mathrm{m}\right)$, with a $1 \mu \mathrm{m}$ thick sacrificial InP layer underneath the slab. An InGaAs etch stop was introduced above the InP substrate, and the active quaternary material was designed to operate at $\lambda=1.55 \mu \mathrm{m}$. The fabrication procedure consisted of electron-beam lithography, followed by two dry- and one wet-etching steps. The etch mask consists of 100-140 nm thick $\mathrm{SiO}_{2}$ or $\mathrm{Si}_{3} \mathrm{~N}_{4}$ layer, deposited at room temperature using a rf sputtering technique. Both masks performed similarly in the etching chemistry, but in this section a $\mathrm{Si}_{3} \mathrm{~N}_{4}$ layer was used as the dielectric mask layer. $120 \mathrm{~nm}$ of poly(methyl methacrylate) (PMMA), electron-beam ( $e$-beam) sensitive resist, was deposited on top of the $\mathrm{Si}_{3} \mathrm{~N}_{4}$ mask layer. A $2 \%$ solution of PMMA (molecular weight $950 \mathrm{~K}$ ) diluted in chlorobenzene was used. The PMMA was baked on a hot plate at $150{ }^{\circ} \mathrm{C}$ for $40 \mathrm{~min}$. $e$-beam lithography was performed using a Leica EBPG 5000+ operating at $100 \mathrm{keV}$, and the dose used to define the patterns in PMMA was $500 \mu \mathrm{C} / \mathrm{cm}^{2}$. By varying the electron dose, one can span the entire design space of the PPC and ABR nanocavities. Upon completing $e$-beam lithography, the PMMA was developed in 1:3 methyl isobutyl ketone: isopropyl alcohol (IPA) PMMA developer and rinsed for $15 \mathrm{~s}$ in IPA. The PMMA patterns were transferred into the $\mathrm{Si}_{3} \mathrm{~N}_{4}$ layer using reactive ion etching (RIE). $20 \mathrm{sccm}$ of $\mathrm{CHF}_{3}$ reactive gas was used and the chamber pressure was kept at 16 mTorr. The rf power used in this RIE step was $90 \mathrm{~W}$ and with a dc bias of $480 \mathrm{~V}$ for $3 \mathrm{~min}$. At the end, the patterns were transferred through the InGaAsP active layer into the InP substrate with an inductive-coupled RIE using an $\mathrm{HI} / \mathrm{H}_{2}$ plasma chemistry. ${ }^{9}$ Finally, the remaining $\mathrm{Si}_{3} \mathrm{~N}_{4}$ mask was removed in $\mathrm{HF}$ and the InGaAsP membrane can be released from the substrate by wet etching in a $4: 1 \mathrm{HCl}$ :water bath at $4{ }^{\circ} \mathrm{C}$. The fabrication procedure is summarized in Fig. 3.

\section{MICROFLUIDIC INTEGRATION OF PHOTONIC CRYSTAL CAVITIES}

Both PPC and ABR lasers can be embedded within polydimethylsiloxane (PDMS) elastomers. In ABRs, this procedure is necessary to maintain the structural integrity of the ring mirrors. For replication molding of PDMS, a photoresist mold was first created using SU8-2015 elastomer to yield a height of approximately $12 \mu \mathrm{m}$. 5:1 GE RTV615 elastomer was then poured onto the mold and partially cured at $80{ }^{\circ} \mathrm{C}$ for $45 \mathrm{~min}$ to create flow channels. Once holes have been 

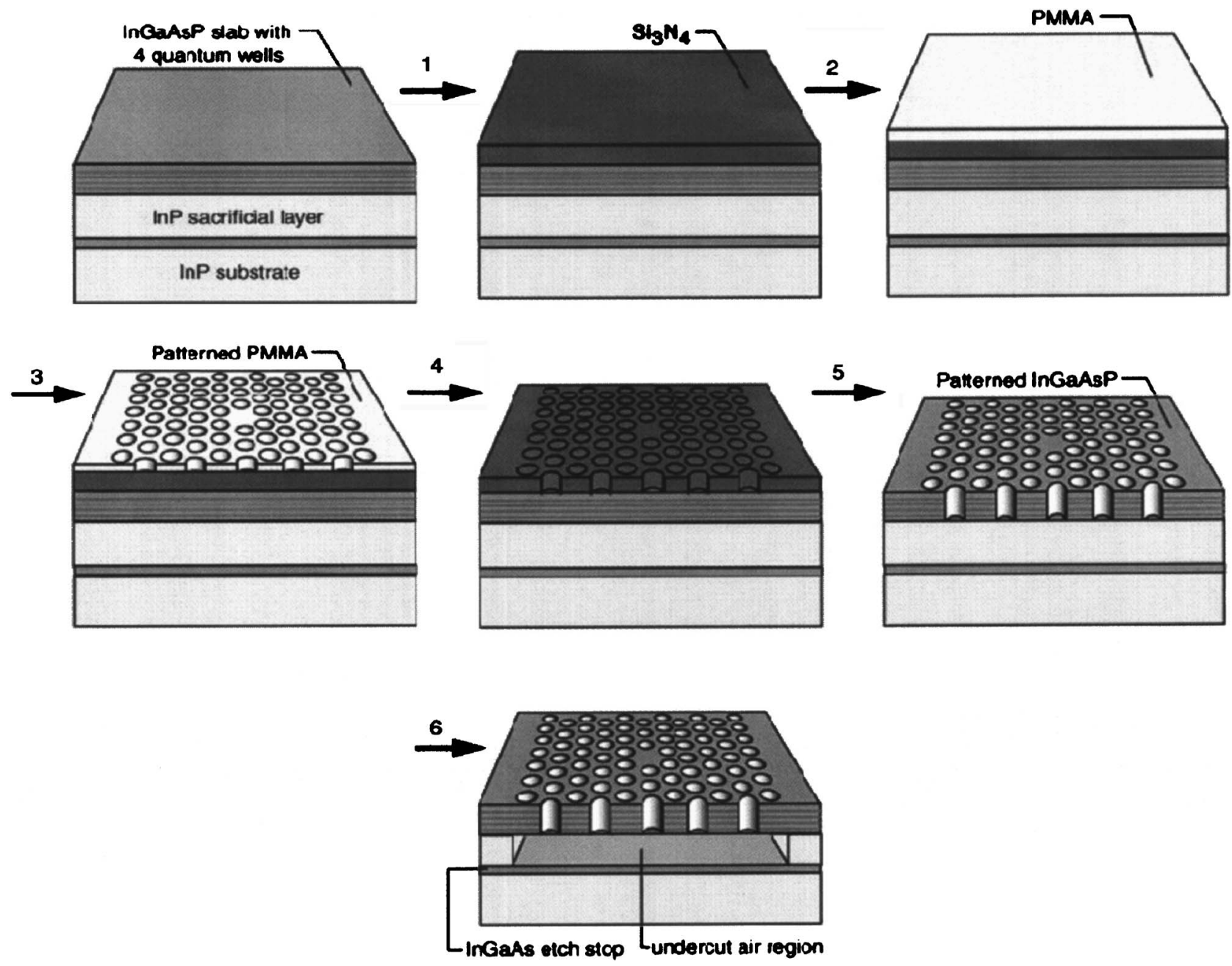

FIG. 3. Fabrication procedure for PPC lasers made in InGaAsP material. rf sputtering (1) is used to deposit dielectric and is followed by deposition of PMMA $e$-beam resist (2). $e$-beam lithography is used to define patterns in PMMA (3) and RIE to transfer them into $\mathrm{Si}_{3} \mathrm{~N}_{4}$ (4). Finally, patterns are etched into InGaAsP using inductively coupled plasma-RIE (5) and the membrane is released from the substrate in $\mathrm{HCl}$ (6). Final structure is a free standing InGaAsP membrane with four quantum wells (thin layers).

punched to access these flow channels, the flow layer can be aligned onto the laser sensor chip so that one flow channel passes over several nanolaser cavities. In order to embed the PPC structure, it must first be coated with PMMA since that polymer does not interfere with the PDMS curing procedure. After baking, the assembly was allowed to cool to room temperature and then a layer of PMMA was spun on top of the assembly to cover the backside of the InP chip.

20:1 GE RTV615 was poured onto the InP wafer assembly and cured. Once the PDMS was fully cured, the assembly was placed into acetone at $95{ }^{\circ} \mathrm{C}$ until the laser structure had lifted off the silicon wafer. Since PDMS is permeable, the acetone seeps through the PDMS layer and dissolves the PMMA that has bonded the laser sources to the silicon wafer. After the two have been separated, the acetone was removed and replaced with IPA. When the laser is freed from the silicon wafer, it is still encapsulated by the PDMS. The PDMS can then be removed from the silicon wafer with the
PPC devices and placed onto a hotplate at $200{ }^{\circ} \mathrm{C}$ to drive off any remaining solvents which may be trapped inside the PDMS.

In order to seal the flow layer to the laser layer, it is necessary to plasma treat the surface of the laser PDMS layer. The PDMS layer was etched with a combination of $\mathrm{O}_{2}$ and $\mathrm{CF}_{4}$ for sealing PDMS to glass. After the plasma treatment the flow layer and laser layer can be aligned, sealed, and then cured overnight at $80^{\circ} \mathrm{C}$. For applications in which it is not too critical to have individual flow channels, a large well, which encompasses the entire laser array, can be fabricated using similar methods. A diagram for the replication molding process of the flow channels in PDMS is shown in Fig. 4.

\section{CHEMICAL SENSING USING PHOTONIC CRYSTAL NANOLASERS}

Fabricated structures were tested at room temperature using the microphotoluminescence approach $(\mu \mathrm{PL})$. A diode 


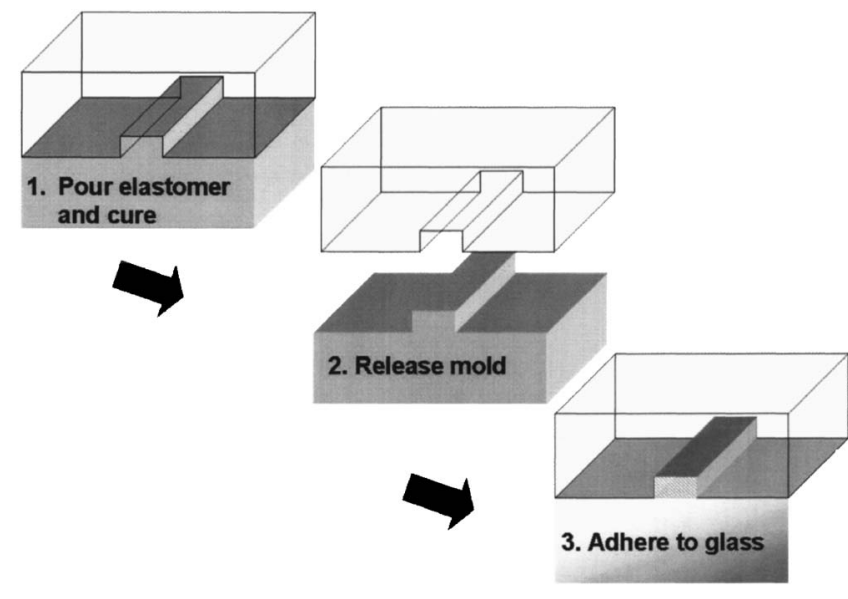

FIG. 4. Replication mold casting process for elastomer flow channels.

laser emitting at $\lambda=830 \mathrm{~nm}$ was used to pump the lasers with $\sim 1 \%$ duty cycles, i.e., $15-30$ ns pulses with $3 \mu$ s periodicity. The pump beam was focused through a $100 \times$ objective lens onto the sample surface to obtain a spot size of about $3 \mu \mathrm{m}$. The emission from the cavities was collected through the same lens, and the spectrum of the emitted signal was analyzed with an optical spectrum analyzer. In order to explore the sensitivity of the lasers on the changes in the refractive index of the environment, fluids with known refractive index in the range $n_{\text {env. }}(1.295,1.335)$ (specified at $\lambda=1.5 \mu \mathrm{m}$ ) with step $\Delta n_{\text {env }}=0.005$ were used.

A $9 \times 8$ cavity [nine rows $(R)$ and eight columns $(C)$ ] laser array was also tested. The results for three different cavities, labeled $R_{6} C_{3}, R_{7} C_{5}$, and $R_{7} C_{3}$ according to their position within the $9 \times 8$ matrix are shown in Fig. 5. Cavities in column $5\left(C_{5}\right)$ have fractional edge dislocation $\mathrm{p} / \mathrm{a}$ $=20 \%$, whereas cavities in column $3\left(C_{3}\right)$ have dislocation of $p / a=10 \%$. Structures in row $6\left(R_{6}\right)$ have lattice constant $a=427 \mathrm{~nm}$, and structures in row $7\left(R_{7}\right)$ have lattice constant $a=454 \mathrm{~nm}$. Figure 5(a) shows the dependence of the frequency of the resonant $H \mathrm{Q}$ mode as a function of the refractive index of environment $\left(n_{\text {env }}\right)$. It can be seen that frequency shifts depend linearly on $n_{\text {env }}$, as predicted by numerical analysis. While the relation between $a / \lambda$ and $n_{\text {env }}$ is useful for comparison between theory and experiments, the relation between $\lambda$ and $n_{\text {env }}$ is of more practical importance. That dependence is shown in Fig. 5(b) and one can see that the emission wavelength depends almost linearly on the refractive index of environment. The sensitivity of the laser emission wavelength on the changes in $n_{\text {env }}$ is $\Delta \lambda$ $=243 . \Delta n_{\text {env }}$ in case of structure $R_{7} C_{3}$, and this is very close to the sensitivity obtained using $3 \mathrm{D}$ finite difference time domain (FDTD) model $\left(\Delta \lambda=266 . \Delta n_{\text {env }}\right)$. In the experiments $n_{\text {env }}$ was changed in steps $\Delta n_{\text {env }}=0.01$. The data points represented by squares in Fig 5(b) correspond to $\Delta n_{\text {env }}=0.005$. It can be seen that they also follow the predicted linear trend, and a redshift of $\approx 1.2 \mathrm{~nm}$ is measured (structure $R_{7} C_{3}$ ) when $n_{\text {env }}$ is changed from 1.315 to 1.32 .
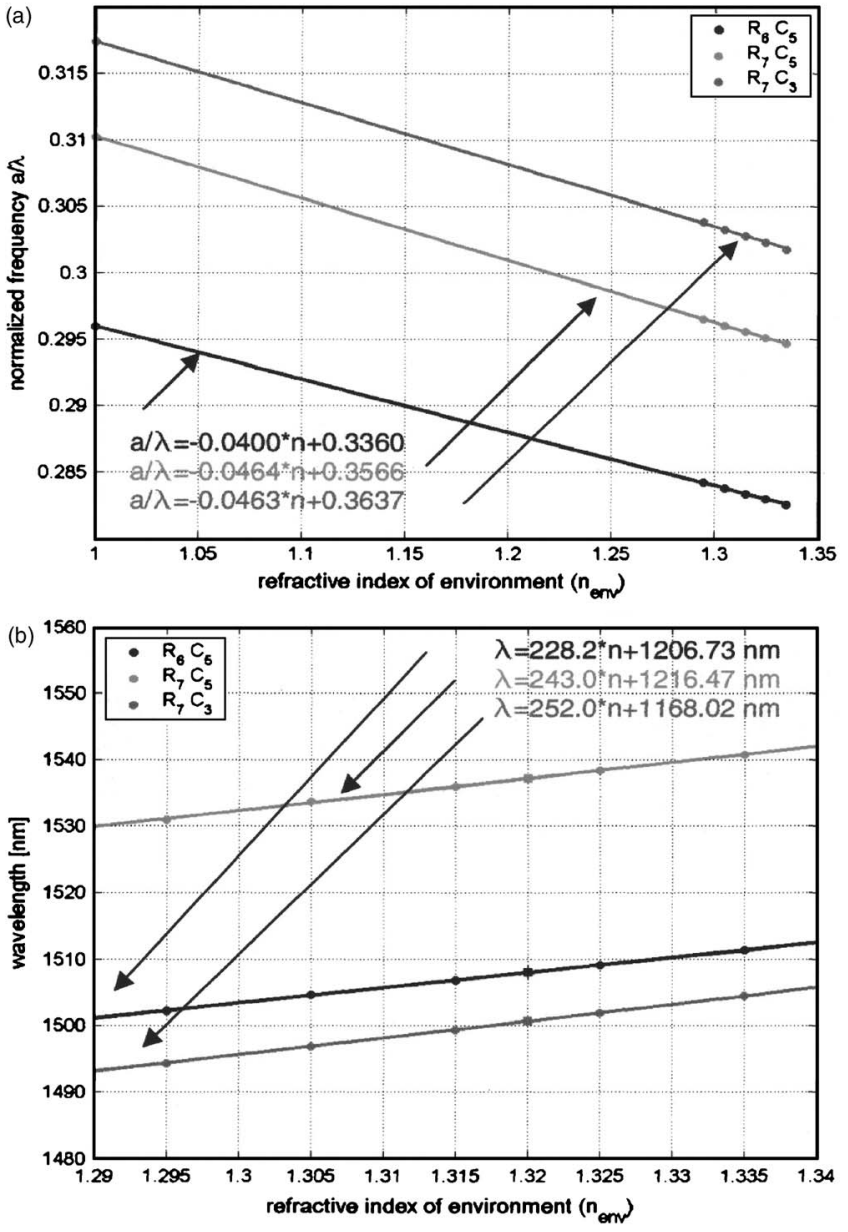

FIG. 5. Dependence of (a) $a / \lambda$ and (b) $\lambda$ of $H Q$ mode on refractive index of the environment $\left(n_{\text {env }}\right)$. Three different cavities, labeled $R_{6} C_{3}, R_{7} C_{5}$, and $R_{7} C_{3}$ were tested.

\section{CONCLUSIONS}

High refractive-index-contrast optical resonators have the potential to radically miniaturize spectroscopic systems. Indeed, the introduction of analytes into porous lasers such as PPC and ABR cavities can result in high sensitivity and femtoliter analyte volumes. Here we have shown that by optimizing the design of these lasers, high sensitivity and small mode volumes are available for chemical analysis.

\section{ACKNOWLEDGMENTS}

The authors wish to acknowledge generous support from DARPA under Contract No. HR0011-04-1-0032, The NIH under Contract No. RFA-CA-05-003, and the AFOSR under Contract No. F49620-02-1-0324.

${ }^{1}$ J. Vuckovic, M. Loncar, H. Mabuchi, and A. Scherer, Phys. Rev. E 65 016608 (2002).

${ }^{2}$ J. Scheuer, W. M. J. Green, G. A. DeRose, and A. Yariv, IEEE J. Sel. Top. Quantum Electron., 11, 476 (2005).

${ }^{3}$ M. Loncar, A. Scherer, and Y. Qiu, Appl. Phys. Lett. 82, 648 (2003).

${ }^{4}$ M. Meier, A. Mekis, A. Dodabalapur, A. Timko, R. E. Slusher, J. D. Joannopoulos, and O. Nalamasu, Appl. Phys. Lett. 74, 7 (1999).

${ }^{5}$ M. Imada, S. Noda, A. Chutinan, T. Tokuda, M. Murata, and G. Sasaki, Appl. Phys. Lett. 75, 316 (1999). 
${ }^{6}$ M. Notomi, H. Suzuki, and T. Tamamura, Appl. Phys. Lett. 78, 1325 (2001).

${ }^{7}$ H. Ryu, S. H. Kwon, Y. J. Lee, Y. H. Lee, and J. S. Kim, Appl. Phys. Lett. 80, 3476 (2002).
${ }^{8}$ S. Noda, M. Yokoyama, M. Imada, A. Chutinan, and M. Mochizuki, Science 293, 1123 (2001).

${ }^{9}$ M. Loncar, M. Hochberg, A. Scherer, and Y. M. Qiu, Opt. Lett. 29, 721 (2004). 